\title{
Liquid-Phase Membrane-Type Shear Horizontal Surface Acoustic Wave Devices
}

\author{
Takashi Kogai ${ }^{1,}$ and Hiromi Yatsuda ${ }^{1,2}$ \\ 'Japan Radio Co., Ltd., 2-1-1 Fukuoka Fujimino-Shi, Saitama 356-8510, Japan \\ ${ }^{2} \mathrm{OJ}$-Bio Ltd., International Center for Life, Newcastle upon Tyne, NE1 4EP, UK
}

(Received January 20, 2014; accepted June 12, 2014)

Key words: membrane, surface acoustic wave, sensor, biosensor, immunosensor

In this paper, we describe a liquid-phase shear horizontal surface acoustic wave (SHSAW) device that is composed of an air-cavity-type SH-SAW device and a membrane on the surface. The air-cavity structure can protect the transducers of the SH-SAW device using unique air cavities. Thus, the air-cavity-type SH-SAW device can be directly dipped into liquids and a liquid can be directly injected onto the surface of the device. In addition, a membrane can be placed directly on the SH-SAW sensor surface and sample liquids can be injected into the membrane. Although a membrane is placed directly on the surface of the device, the frequency response of the SH-SAW devices cannot be degraded. In addition, when liquids are injected, the SH-SAW device with a membrane can work as well as a conventional SH-SAW device without membranes.

\section{Introduction}

Immunoassay-based biosensors have been widely investigated because they can provide rapid and high-selectivity detection. Currently, immunochromatographic lateral flow strip tests have been commonly used as a one-step test that facilitates low-cost, rapid identification of various analytes at the point of care. On the other hand, shear horizontal surface acoustic wave (SH-SAW) sensors have been attracting a wide range of attention because of their various advantages, such as real-time and label-free detection of mechanical property and electrical property in the liquid phase. Various applications have been demonstrated using SH-SAW sensors, ${ }^{(1-10)}$ including the chemical detection of conductivity and permittivity ${ }^{(1-3)}$ and the biological detection of mass density and viscosity. ${ }^{(4-7)}$ It has been shown that SH-SAW sensor systems can detect antigens in a nanomole range and provide quantitative results. ${ }^{(6)}$

In this paper, a liquid-phase SH-SAW device with a membrane on the surface is described. The membrane can provide some attractive functions, such as keeping sample

${ }^{*}$ Corresponding author: e-mail: kogai.takashi@jrc.co.jp 
solutions on the sensor surface, filtering the specimen (separation of blood plasma), and having gold-conjugated antibodies inside. The membrane techniques can be very attractive for liquid-phase SH-SAW biosensors.

\section{Materials and Methods}

\subsection{SH-SAW delay-line device and electrical reader}

A liquid-phase SH-SAW delay-line device is shown in Fig. 1. The delay line has a transmitting interdigital transducer (IDT), receiving IDT, and a propagation area between them. The propagation areas are covered with a gold film. Once antibodies have been immobilized onto the gold surface, the SH-SAW device can act as an immunosensor. The transmitting and receiving IDTs are placed at each end, and the center-to-center distance between them is $9 \mathrm{~mm}$. A center frequency of $251.5 \mathrm{MHz}$ was designed using a wavelength of $20 \mu \mathrm{m}$ on a $36^{\circ}$ Y-cut quartz substrate. The device has air cavities above the IDTs that are composed of epoxy walls that surround the IDTs and glass lids. The walls of $60 \mu \mathrm{m}$ thickness and $50 \mu \mathrm{m}$ height were built by a photolithography technique using a thick epoxy photoresist, SU-8 from MicroChem Corp. A glass lid of $4.4 \times 3.2 \times$ $0.2 \mathrm{~mm}^{3}$ size was placed on the wall and was attached using an epoxy adhesive. Since the IDTs are protected from liquids, the devices can be directly dipped into a liquid and a liquid can be directly injected onto the surface of the device. The SH-SAW device is assembled onto a printed circuit board. After wire bonding, the wires are protected using epoxy adhesive. ${ }^{(6)}$

The propagating SH-SAW on the metallized surface can be affected by the mechanical properties of the liquid; ${ }^{(8-10)}$ thus, the velocity and attenuation of the SHSAW can be changed. To measure the phase and amplitude changes of the SH-SAW, we designed a printed circuit board of $120 \times 60 \mathrm{~mm}^{2}$ size containing a signal generator, a measurement circuitry for the phase and amplitude differences, a burst circuit to eliminate unwanted electromagnetic feed-through signals, and an $\mathrm{A} / \mathrm{D}$ converter with an internal microprocessor. ${ }^{(7)}$ A prototype electric reader of $140 \times 100 \times 40 \mathrm{~mm}^{3}$ size was achieved. The velocity and attenuation changes of SH-SAWs are calculated using the phase and amplitude changes that are measured usnig the electric reader. The block diagram of the SH-SAW sensor setup is shown in Fig. 2 and a photograph of the sensor setup is shown in Fig. 3.

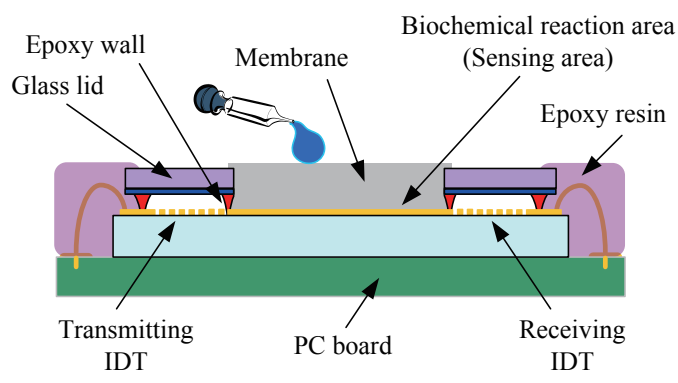

Fig. 1. (Color online) Diagram of SH-SAW sensor device with membrane. 

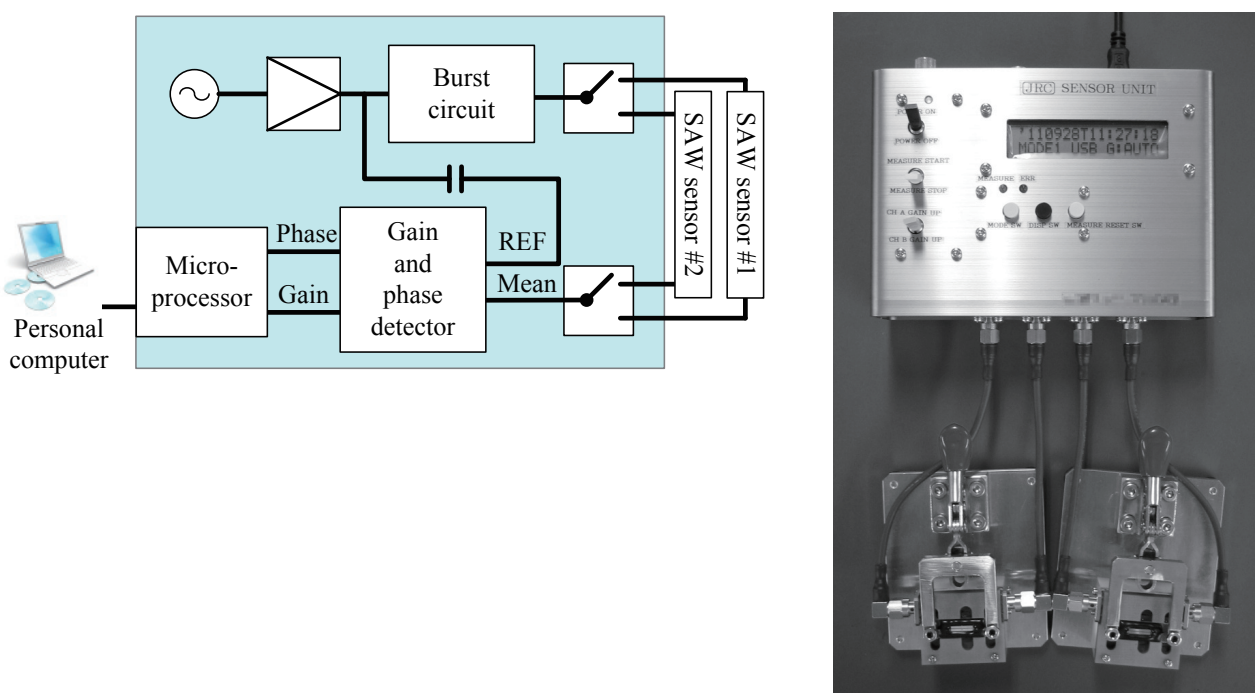

Fig. 2 (left). (Color online) Block diagram of SH-SAW biosensor detection circuit.

Fig. 3 (right). Photograph of SH-SAW biosensor setup.

\subsection{Membrane-type SH-SAW device}

A unique membrane-type SH-SAW device is shown in Fig. 4. A membrane of $9 \mathrm{~mm}$ length and $2.2 \mathrm{~mm}$ width is placed on the sensing area of the SH-SAW device. When liquid samples are injected into the membrane, the surface of the SAW device can be wetted. In this paper, a conjugate pad membrane and an absorbent pad membrane were tested. The characteristics of the membranes are shown in Table 1. The conjugate pad membrane can be thin. The sample liquids can flow through the membrane with a high flow rate. Those are the advantages of the conjugate pad membrane. On the other hand, the absorbent pad membrane is thick. The membrane can hold a large volume of sample liquids. Those are the advantages of the absorbent pad membrane. The water absorption values are shown in Table 1; the weight was measured using an electronic balance before and after dipping the $1 \mathrm{cc}$ of membrane in pure water. In the experiments, the sample volumes of $30 \mu \mathrm{l}$ for a conjugate pad and $50 \mu \mathrm{l}$ for an absorbent pad are injected, and the sample volume of $20 \mu \mathrm{l}$ for no membrane is injected into the SH-SAW device.

\section{Experimental Results and Discussion}

\subsection{Frequency responses of membrane-type $\mathrm{SH}-\mathrm{SAW}$ devices}

The frequency responses of the SH-SAW delay-line devices are shown in Fig. 5. Figure 5(a) shows the frequency responses of the SH-SAW device without a membrane, and Fig. 5(b) shows those of the SH-SAW device with a conjugate pad membrane. In our experiment, the SH-SAW propagation loss at the epoxy wall was $0.8 \mathrm{~dB} /$ wavelength. ${ }^{(6)}$ The insertion losses of $22.4 \mathrm{~dB}$ in air and $44.5 \mathrm{~dB}$ in water, including the propagation loss of 


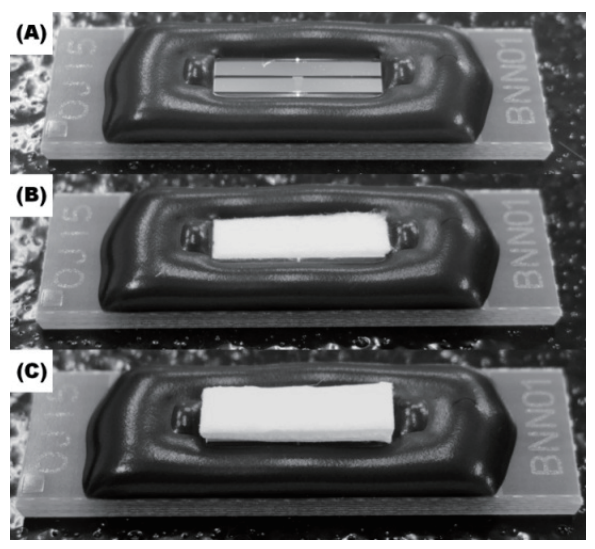

Fig. 4. Photographs of membrane-type SH-SAW sensor: (a) without membrane, (b) with conjugate pad membrane, and (c) with attached absorbent pad membrane.

Table 1

Characteristics of membranes.

\begin{tabular}{lcccc}
\hline Type & Material & $\begin{array}{c}\text { Thickness } \\
{[\mathrm{mm}]}\end{array}$ & $\begin{array}{c}\text { Membrane weight } \\
{\left[\mathrm{mg} / \mathrm{cm}^{2}\right]}\end{array}$ & $\begin{array}{c}\text { Water absorbance } \\
{\left[\mathrm{mg} / \mathrm{cm}^{2}\right]}\end{array}$ \\
\hline Conjugate pad & Bound glass fiber & 0.36 & 5.6 & 49.1 \\
Absorbent pad & Cotton linter & 1.45 & 52.6 & 172.1 \\
\hline
\end{tabular}

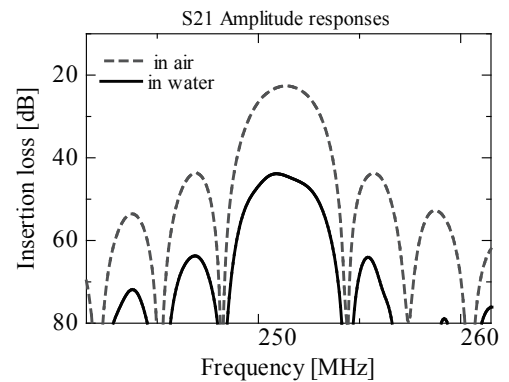

(a)

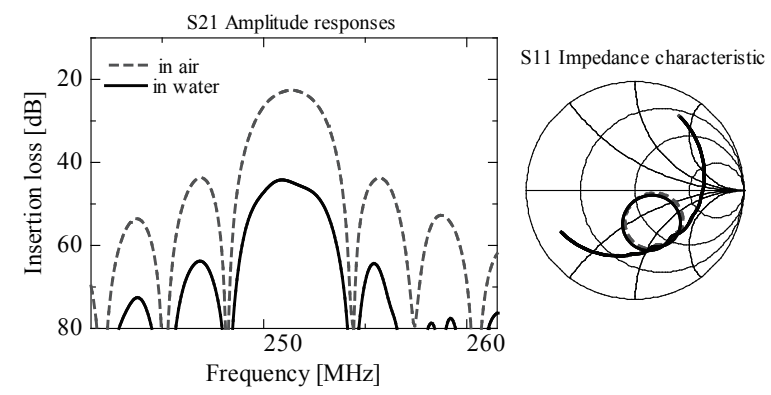

(b)

Fig. 5. Frequency responses of SH-SAW devices: (a) without membrane and (b) with conjugate pad membrane. 
$4.8 \mathrm{~dB}(0.8 \mathrm{~dB} /$ wavelength $)$ at the epoxy walls, were obtained. ${ }^{(6)}$ The insertion losses of $22.6 \mathrm{~dB}$ in air and $44.9 \mathrm{~dB}$ in water were obtained for the SH-SAW device with a conjugate pad membrane and an absorbent pad, as well as for that without a membrane. The insertion losses of the devices are shown in Table 2. The membrane was placed on the sensing area of $9 \mathrm{~mm}$ (450 wavelength) but the insertion loss changes were less than 0.2 $\mathrm{dB}$ in air and less than $0.4 \mathrm{~dB}$ in water. It is clear that the membranes cannot degrade the frequency responses of SH-SAW devices.

\subsection{Real-time responses of velocity changes of SH-SAWs with membranes}

To evaluate the basic characteristics of the SH-SAWs with membranes, glycerolwater mixture liquids of different viscosities were used. Glycerol-water mixture liquids of 1,5 , and $10 \%(\mathrm{w} / \mathrm{w})$ concentrations were injected into the devices without membranes and with a conjugate pad membrane. Real-time responses of the velocity changes of the SH-SAWs are shown in Fig. 6: Fig. 6(a) for the SH-SAW without a membrane and Fig. 6(b) for that with a conjugate pad membrane. After the sample liquid was injected into the membrane, the surface of the SH-SAW device was immediately wetted by the sample liquid through the membrane. The velocity changes of the SH-SAW are shown in Fig. 7 as a function of glycerol concentration. The SH-SAW device with a conjugate pad membrane can provide a similar result as that for the SH-SAW device without

Table 2

Insertion losses of SH-SAW devices.

\begin{tabular}{lcc}
\hline Name & In air [dB] & In water [dB] \\
\hline (A) Without membrane & 22.64 & 44.62 \\
(B) With conjugate pad & 22.65 & 44.99 \\
(C) With absorbent pad & 22.66 & 44.86 \\
\hline
\end{tabular}

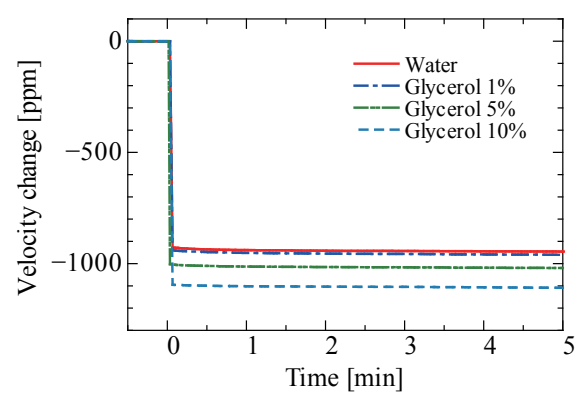

(a)

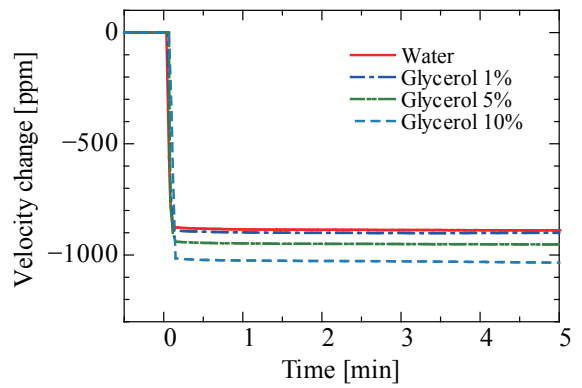

(b)

Fig. 6. (Color online) Fractional velocity changes of SH-SAW with membrane with respect to glycerol concentration: (a) without membrane and (b) with conjugate pad membrane. 


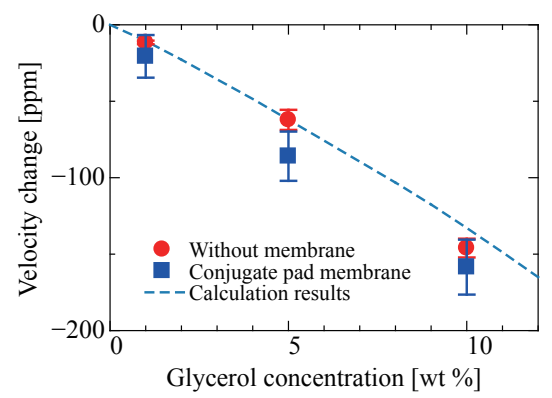

Fig. 7. (Color online) Velocity changes vs glycerol concentration.

membranes and as the calculation result. ${ }^{(8)}$ A small difference was observed in Fig. 7. The reason may be a viscosity change of the liquid owing to the fact that the membrane diffused into the liquid.

\subsection{Binding characteristics of SH-SAW devices with membranes}

To evaluate the binding characteristics of the SH-SAW devices with membranes, phosphate-buffered saline (PBS) with different concentrations of human serum albumin (HSA) antigens was injected into the SH-SAW devices. As the HSA antigens can be bound with a gold surface of the SH-SAW devices, the SH-SAW velocity can be changed. A series of PBS-diluted HSA antigen solutions of concentrations from $0,0.15,1.5$, and $15 \mu \mathrm{M}$ were directly injected into the gold surface of the device or to the membrane on the device. After the PBS solution with HSA antigens was injected into the SHSAW devices, the real-time velocity changes that occurred for SH-SAWs without the membrane, with the conjugate pad membrane and with the absorbent pad membrane are shown in Figs. 8(a)-8(c), respectively. After a 2-min incubation, the velocity changes for direct binding of HSA antigens to the gold surface of the SH-SAW devices that occurred are shown in Fig. 9.

\subsection{Membrane functions}

In the case of a hard-type membrane, such as nitrocellulose membrane, there can be a layer between the membrane and the chip surface as shown in Fig. 10(a). The SHSAW cannot be affected by the membrane. In the case of a soft type membrane, such as cellulose-fiber membrane, there might be a mixture layer of the membrane and the liquid on the chip surface as shown in Fig. 10(b), the viscosity of the mixture layer might be slightly different from the viscosity of the liquid. So the velocity and amplitude of the SH-SAW might slightly change due to the membrane. However, the reference channel technique can compensate for this effect. The SH-SAW biosensors with a membrane can provide some additional attractive functions: holding the sample on the chip surface, delivering the sample to the chip surface from the sample injection area, filtering the specimen (separation of blood plasma), and keeping the gold-conjugated antibodies in the membrane. 


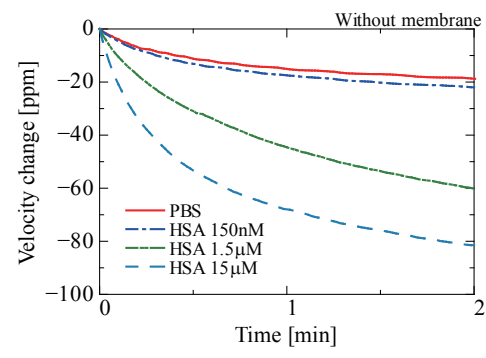

(a)

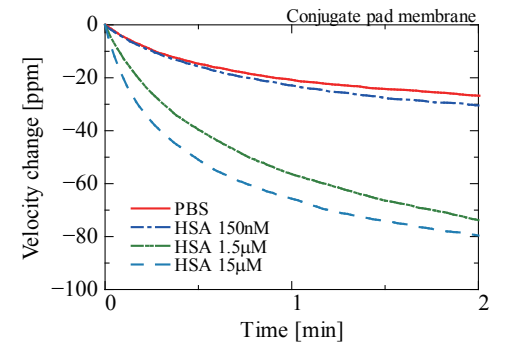

(b)

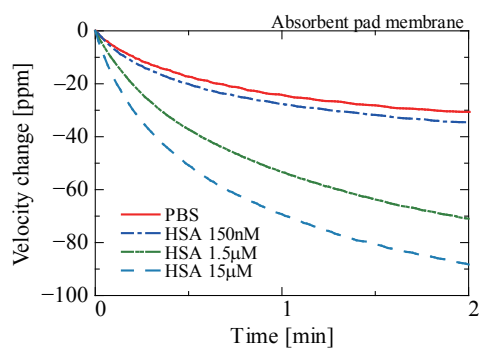

(c)

Fig. 8. (Color online) Fractional velocity changes of SH-SAW devices with respect to HSA antigen concentrations: (a) without membrane, (b) with conjugate pad, and (c) with absorbent pad.
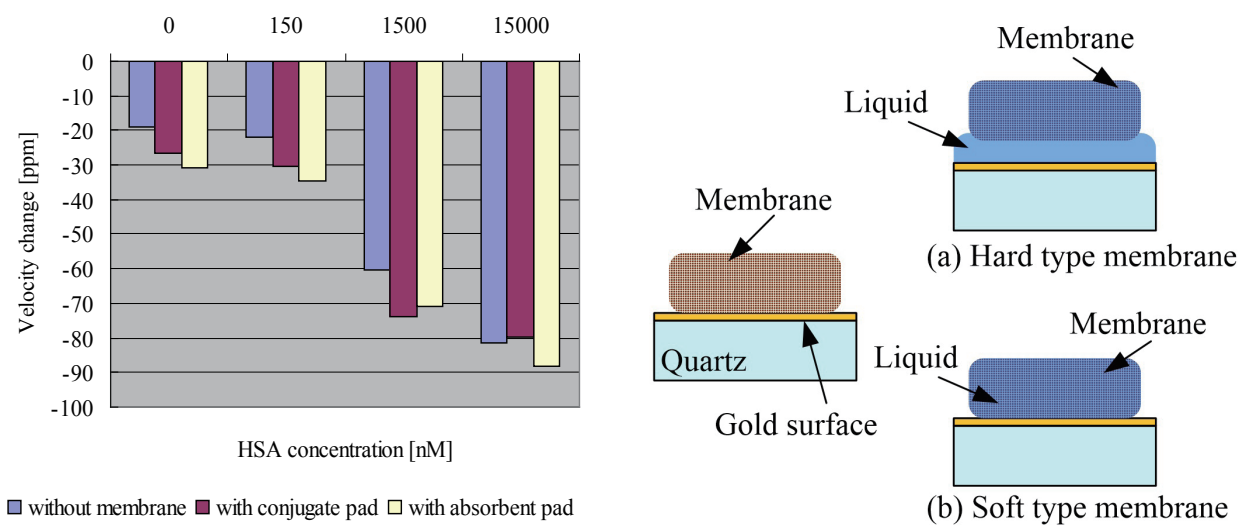

Fig. 9 (left). (Color online) Velocity changes of SH-SAWs vs HSA antigen concentration.

Fig. 10 (right). (Color online) Diagram of membrane function on sensing area of SH-SAW device.

\section{Conclusions}

This paper is the first report on liquid-phase SH-SAW devices with a membrane on the surface. No degradation of the frequency responses of SH-SAW devices with a membrane was observed. After the sample solution was injected into the membrane, the surface of the SH-SAW device could be immediately wetted by the sample liquid through the membrane. We have introduced a new concept of membranes on SH-SAW biosensors. The membrane techniques is attractive for liquid-phase SH-SAW biosensors that have a gold surface covered with antibodies. The SH-SAW biosensors with a membrane can provide some additional attractive functions: holding the sample on the chip surface, delivering the sample to the chip surface from the sample injection area, filtering the specimen (separation of blood plasma), and keeping the gold-conjugated antibodies in the membrane. The unique membrane-type SH-SAW sensor devices are suitable for easy-to-use one-step biosensors. 


\section{Acknowledgements}

We are deeply grateful to the staff members of OJ-Bio Ltd., in the UK for their continuing support and constant encouragement of discussions and experiments.

\section{References}

1 J. Kondoh, Y. Okiyama, S. Mikuni, Y. Matsui, M. Nara, T. Mori and H. Yatsuda: Jpn. J. Appl. Phys. 35 (1996) 3093.

2 T. Kogai, H. Yatsuda and S. Shiokawa: Jpn. J. Appl. Phys. 47 (2008) 4091.

3 K. Kano, T. Kogai, N. Yoshimura, H. Yatsuda, J. Kondoh and S. Shiokawa: Jpn. J. Appl. Phys. 51 (2012) 07GC20.

4 T. Moriizumi, Y. Unno and S. Shiokawa: Proc. IEEE Int. Frequency Control Symp., 1987, p. 579 .

5 E. Berkenpas, S. Bitla, P. Millard and M. Pereira da Cunha: IEEE Trans. Ultrason. Ferroelectr. Freq. Control 51 (2004) 1404.

6 T. Kogai, N. Yoshimura T. Mori, and H. Yatsuda: Jpn. J. Appl. Phys. 49 (2010) 07HD15.

7 M. Goto, O. Iijima, T. Kogai and H. Yatsuda: Proc. IEEE Ultrasonics Symp., 2010, p. 736.

8 M. Goto, H. Yatsuda and J. Kondoh: Proc. IEEE Ultrasonics Symp., 2012, pp. 2110.

9 M. Goto, H. Yatsuda and J. Kondoh: Jpn. J. Appl. Phys. 52 (2013) 07HD10.

10 J. Kondoh, K. Saito, S. Shiokawa and H. Suzuki: Proc. IEEE Ultrasonics Symp., 1995, p. 445. 